\title{
Effect of Isometric Exercise on Blood Pressure in Pre-Hypertensive and Hypertensive Individuals: Protocol for a Systematic Review and Meta-analysis of Randomized Clinical Trials
}

\section{Patricia Caetano Oliveira}

Institute of Cardiology of Rio Grande do Sul/Univsersity Foundation of Cardiology

Alexandre Machado Lehnen ( $\square$ amlehnen@gmail.com )

Institute of Cardiology of Rio Grande do Sul/University Foundation of Cardiology

https://orcid.org/0000-0002-5912-8020

\section{Gustavo Waclawovsky}

Institute de Cardiology of Rio Grande do Sul/University Foundation of Cardiology

\section{Protocol}

Keywords: pre-hypertension, hypertension, blood pressure, isometric exercise

Posted Date: January 19th, 2021

DOl: https://doi.org/10.21203/rs.3.rs-148325/v1

License: (c) (1) This work is licensed under a Creative Commons Attribution 4.0 International License.

Read Full License 


\section{Abstract}

\section{Background}

Systemic arterial hypertension (HTN) is the leading risk factor of cardiovascular disease death. Lifestyle changes are key for the prevention and management of HTN. Regular aerobic exercise training is recommended as part of the management of HTN; and dynamic resistance exercise should be prescribed as an adjuvant to aerobic training. Recent evidence points to potential benefits of isometric resistance training in reducing blood pressure (BP). Yet, the hypotensive effect of isometric exercise in prehypertensive and hypertensive individuals is not fully understood. Thus, we will examine the effect of isometric exercise in pre-hypertensive and hypertensive individuals through a systematic review and meta-analysis.

Methods

Our systematic review study will include randomized clinical trials (RCTs) selected from the electronic databases MEDLINE (PubMed), Cochrane, LILACS, EMBASE and PEDro published in English, Spanish and Portuguese language. We will follow the PRISMA guidelines and PICOS framework. Our search will involve studies with both male and female participants aged 18 years or more diagnosed with prehypertension or HTN performing one session of isometric exercise (acute effect) or isometric exercise training (chronic effect) compared to a control group (no exercise). We will use Cochrane Risk of Bias 2 (RoB 2) tool to evaluate the quality of the studies and RStudio software (v1.3.959 for Windows) for statistical analyses.

\section{Discussion}

A meta-analysis of a homogeneous sample of pre-hypertensive and hypertensive individuals involving isometric handgrip exercise alone can further support previous findings and improve our understanding and recommendations for the management of these populations.

Systematic review registration

The study protocol for this review was registered in the International Prospective Register of Systematic Reviews (PROSPERO) (CRD 42020213081).

\section{Background}

Systemic arterial hypertension (HTN) prevalence is estimated at $30 \%$ of worldwide population, affecting nearly 1.4 billion adults $(1,2)$. The World Health Organization (WHO) has projected this number will escalate to 1.6 billion people by $2025(1,3)$. This is a concerning scenario because $10 \mathrm{mmHg}$ increase in systolic blood pressure (SBP)/diastolic blood pressure (DBP) levels has been strongly associated with an increase in the rate of ischemic heart disease deaths $(4,5)$. HTN represents the leading risk factor for 
cardiovascular diseases (13\%) as compared to other risk factors including smoking (9\%), high blood glucose (6\%), physical inactivity (6\%) and excess body weight (5\%) (6).

The practice of regular aerobic exercise has been traditionally recommended as the first-line nonpharmacological management of cardiovascular diseases. Dynamic resistance exercise involving joint movement is recommended as an adjuvant intervention to aerobic training (7-9). Interestingly, isometric resistance exercise (not involving joint movement) is apparently effective in reducing blood pressure (BP) levels as evidenced in meta-analyses published over the last 10 years (10-16). A meta-analysis by Cornelissen et al. (2013) evaluated the hypotensive effect of aerobic, dynamic resistance and isometric resistance training. They found that isometric training elicited BP reductions greater than those seen in the control group and with other exercise modalities as well (12). Yet, this meta-analysis included only four studies of isometric training.

Drawing from the findings of studies published in the literature, the Canadian Cardiovascular Society recommends isometric handgrip training as an adjuvant to dynamic resistance training for normotensive or stage 1 hypertensive individuals (17). Likewise, the American Heart Association also endorses isometric training as an adjuvant BP-lowering strategy (18).

Despite the significant number of studies supporting these recommendations, the samples of participants from the studies included in these meta-analyses are not fully representative of prehypertensive or hypertensive populations $(10-12,19)$. They are usually heterogeneous samples of normotensive, pre-hypertensive and/or hypertensive individuals, making it difficult to generalize the results for each group. Moreover, some studies involved participants with other comorbidities that might have affected their results. It is crucial to examine restricted samples of hypertensive individuals as they show higher BP levels in response to exercise and require longer recovery intervals between resistance exercise sets compared to normotensive individuals (20). In addition, most meta-analyses $(10,12,13,16$, 19) evaluated the effect of isometric exercise from studies with interventions of combined exercise involving upper and lower limbs, and since the amount of active muscle mass involved is different hemodynamic responses to this type of exercise can be also different $(21,22)$. To our understanding, if handgrip exercise alone is proven effective for the prevention and management of HTN it could be prescribed as an alternative exercise approach for those who are not able to perform isometric leg exercise due to physical limitation, peripheral artery disease or any other limiting condition.

Recently published meta-analyses $(14,15)$ have shown improved methodological quality. However, Jin et al. (2017) evaluated a small number of studies (only seven) in their meta-analysis (four with hypertensive individuals; two with normotensive individuals; and one with pre-hypertensive individuals) (14). Loaiza et al. (2020) conducted a more consistent metanalysis with 11 articles of pre-hypertensive and hypertensive individuals; yet, three randomized controlled trials (RCTs) (23-25) did not use a control group for comparison of the analyses (15).

The hypotensive effect is a relevant event in both a single session of isometric exercise and exercise training over time, so it is valuable to conduct a consistent meta-analysis with special focus on pre- 
hypertensive and hypertensive populations. To the best of our knowledge, there is no meta-analysis of studies investigating the acute effect of handgrip exercise on BP and they have examined only the chronic effect of this type of exercise. Hence, we developed a study protocol for a systematic review and meta-analysis including RCTs evaluating BP-reducing effect of isometric exercise or training in prehypertensive or hypertensive individuals.

\section{Methods}

This study follows the guidelines of the Preferred Reporting Items for Systematic Reviews and Metaanalyses (PRISMA) (26). The protocol for the systematic review and meta-analysis was registered in the International Prospective Register of Systematic Reviews (PROSPERO) (www.crd.york.ac.uk/PROSPERO/, CRD 42020213081 and registration date 09/10/2020). The Figure summarizes the study design.

\section{Eligibility criteria}

The PICOS framework was used for structuring the design of the study, research question and search as follows: Population (individuals aged $\geq 18$ years diagnosed with pre-HTN or HTN); Intervention (one session of isometric resistance exercise or isometric handgrip training); Comparison (isometric resistance exercise or training versus [no exercise or training] control group); O utcome (SBP and DBP measurements by auscultation, oscillometry or 24-hour ambulatory BP monitoring); Study (RCTs).

Inclusion criteria for this review study include "prehypertension" and/or "hypertension" in the title and/or abstract used to describe the main characteristic of the study sample. The main body of the article must have a clear description of the study population (adults) and diagnosis of HTN: sustained high BP levels and/or use of antihypertensive drugs together with a medical history and/or skilled medical assessment. The criteria for "intervention" include a study with two or more arms, either isometric resistance exercise or training using handgrip as "comparator" versus a control group (no session of exercise or physical training, or receiving only primary care, usual care, care while on a waiting list etc.). For the purpose of conducting subgroup analyses, all exercise frequencies, intensities and times will be included. Only RCTs of prehypertensive or hypertensive individuals, defined in each RCT (overall, sustained high BP levels and/or use of antihypertensive drugs together with a medical history and/or skilled medical assessment), will be selected for the meta-analysis.

\section{Inclusion and exclusion criteria}

We will select all studies of individuals aged 18 or more with HTN measured using auscultation, oscillometry or 24-hour ambulatory BP monitoring (ABPM) involving isometric handgrip exercise or training as an intervention. Studies involving other interventions associated with exercise but with a clearly defined isometric resistance group and a control group (no exercise) will be fully reviewed for inclusion. Studies with participants taking antihypertensive medications will be eligible when medication was introduced either $\geq 4$ weeks before the exercise/training intervention or during the intervention and data analysis. 
Studies with individuals taking medication other than antihypertensive drugs or concomitant dietary (or supplement) interventions as well as review studies, studies with animal experimentation and studies that involve any condition other than pre-HTN or HTN will be excluded. Studies with similar characteristics published in different journals will be carefully reviewed and excluded if considered "duplicated publication."

\section{Search strategy}

A prior search was conducted in the Medline database via PubMed to ascertain whether the research question of our review meets the FINER criteria (feasible, interesting, novel, ethical and relevant). A search strategy for RCTs was then developed to be undertaken by two independent reviewers (PCO and GW) in the databases recommended in the Cochrane Handbook for Systematic Reviews of Interventions (27): Medline, EMBASE (database of published European literature) and Cochrane databases (for access to trials that may not be indexed in MEDLINE and EMBASE). To broaden our search results, we will conduct searches for the Latin America literature through LILACS (Latin American and Caribbean Health Sciences Literature / Virtual Health Library [VHL]). Given that the intervention of interest may also be part of physical therapy rehabilitation programs, our searches will also be conducted in the Physiotherapy Evidence Database (PEDro, www.pedro.org.au). To minimize any publication bias, searches will also be undertaken on online gray literature including OpenGrey (www.opengrey.eu) and the Brazilian Coordination for the Improvement of Higher Education Personnel (CAPES) Bank of Theses and Dissertations (www.catalogodeteses.capes.gov.br). For unpublished ongoing trials, our searches will be undertaken in clinical trial registries: Brazilian Clinical Trials Registry (ReBEC, www.ensaiosclinicos.gov.br), Clinical Trial.gov (www.clinicaltrials.gov) and WHO International Clinical Trials Registry Platform (ICTRP, www.who.int/ictrp/en/). We will gather data through careful review of the articles retrieved and will contact authors by email to obtain any additional information as required. Articles in Portuguese, English and Spanish language with no date of publication limits will be eligible for inclusion. Upon completion of the review, we will undertake an additional search of all databases and registry platform to ensure the inclusion of the most recent studies.

The main search terms will include "hypertension," "exercise," "isometric exercise" and "blood pressure" (Table 1). The search terms for the study design (randomized clinical trial, RCT) will be entered in the databases MEDLINE (28) and EMBASE (29) for more accurate and sensitive searches (Table 1). The two study reviewers (PCO and GW) will work independently selecting the studies after an initial screening of titles and abstracts. When there will be no sufficient information in the abstract, the reviewers will retrieve and read the full text of the article. Any discrepancies will be resolved through a consensus discussion and, if necessary, any disagreements on the inclusion criteria will be resolved by a third reviewer (AML).

\section{Data extraction and management}

After a complete search in each database, all articles retrieved will be exported as ".ris" or ".enib" files and imported into EndNote X9 arranged in folders by search platform, exclusion criteria and eligibility. All duplicates will be removed using EndNote X9 Find Duplicates command (Menu All references / 
References / Find duplicates). Our reviewers (PCO and GW) will use a search tool (Search, Title OR $A b s t r a c t$ ) to check for any remaining duplicates. In the next step, they will read the full text of all eligible studies. If they are considered relevant, the main data of the studies selected will be extracted and compiled in a pre-structured database in Excel 2010 for Windows: 1) study (authors, journal, year of publication, intervention, characteristics of exercise session or training); 2) participants (age, gender, body mass index [BMI], medical condition); and 3) methods (randomization, blinding) and outcome (sample size, mean and standard deviation at baseline and post-exercise session or training). For eligible studies with results presented in graphs, we will contact the authors by email to obtain this data or use GetDate Graph Digitizer 2.26 to extract the data. For studies evaluating BP at different time points, we will independently compare baseline measurements with results at each time point. Then our database will be formatted and imported into RStudio for data analyses.

\section{Risk of bias}

The risk of bias in RCTs will be assessed using Cochrane RoB 2 tool (www.riskofbias.info/) included in Cochrane Handbook $(27,30,31)$. The assessment is based on a set of six domains of bias and judgement can be low, high or unclear risk of bias: randomization sequence generation; allocation concealment; blinding of participants and personnel; blinding of outcome assessment; incomplete outcome data; and selective reporting. If participants are not blinded because blinding is not feasible for exercise intervention (one session of exercise or training), all studies will be classified as high risk of bias in the "blinding of participants and personnel" domain. No study will be excluded based on the risk of bias assessment. The risk of bias will be analyzed for the primary outcomes of interest in our review.

\section{Risk of overall bias in systematic reviews}

We will evaluate the strength of the evidence using the Grading of Recommendations Assessment, Development and Evaluation tool (GRADE; www.gradepro.org) (32). This tool evaluates confidence in estimates of paired effects as well as classifies treatment in a network meta-analysis: study design; methodological limitations (risk of bias); inconsistency; indirectness of evidence; imprecision; publication bias; magnitude of effect; dose-response gradient; and residual confounders (32).

\section{Statistical analysis strategy}

We will conduct separate analyses for the acute effect of isometric resistance exercise and the chronic effect of isometric handgrip training. All measures of effect will be presented as mean differences and their $95 \%$ confidence intervals ( $95 \% \mathrm{Cls}$ ) between exercise or training arms versus a (no exercise) control group. When data is reported in different units, the standardized mean difference will be used as it expresses the size of the intervention effect in each study relative to the variability observed in that study, together with their $95 \% \mathrm{Cl}$. Mean differences or standardized mean differences will be pooled using a fixed- or random-effects model which is appropriate. A random-effects model will be used if studies do not have sufficient similarities to warrant a fixed-effects model and/or the data is reported in different units. 
The summary estimate and confidence interval of the random effect refer to the center of the distribution of intervention effects, but do not describe the width of the distribution. Since the confidence interval from random effects refer to uncertainty in the location of the mean of systematically different effects in the studies, we will consider calculated values for a prediction interval (variation in treatment effects over different settings including what treatment effect is to be expected in future patients) (33) (Chart 1S, supplementary material).

Heterogeneity (percentage of the variability in effect estimates) will be assessed using the $I^{2}$ statistic and Cochran $Q$ test for each pairwise comparison $(27,34)$ (Chart 2S; supplementary material). If heterogeneity is found $(p<0.05)$ for potential effect modifiers, it will be tested using subgroup analysis or metaregression (27). Potential confounders including age, gender, BMI, intervention duration (minutes OR weeks), exercise frequency per week, intervention intensity (low, moderate and high) will be assessed a priori.

A funnel plot will be constructed to assess publication bias if 10 or more studies are eligible to be included in the meta-analysis. All statistical tests will be two-tailed and the significance will be set at $\mathrm{p}<$ 0.05. Data modelizations will be performed with RStudio (version 1.3.959) using R package meta (version 3.6.1 for Windows) (https://www.r-project.org/). Chart 3S summarizes the main RStudio script (supplementary material) (database: META_ISOMETRICpas).

\section{Discussion And Conclusions}

A good number of studies have reported beneficial effects of isometric handgrip exercise on BP (10-16). Yet, after a comprehensive evaluation of the published meta-analyses we found that subgroup analyses are seldom conducted $(10-12,19)$, and when they are performed (13-16), some confounding effects are present such as combined upper- and lower-limb exercises $(10,12,13,16,19)$ that may potentially interfere with hemodynamic responses (21).

Bearing in mind the clinical significance of the hypotensive effect of isometric exercise training, we believe it requires further examination in pre-hypertensive and hypertensive individuals. It is also important to evaluate potential hypotensive effects of isometric handgrip or leg exercise alone (leg press, free squats etc.) as some people are not able to perform certain types of exercises due to physical limitations or other reasons.

In conclusion, understanding the potential BP benefits of isometric exercise alone is important as it may provide alternative exercise options for the management of pre-hypertensive and hypertensive populations. A meta-analysis of a homogeneous sample of pre-hypertensive and hypertensive individuals involving isometric handgrip exercise alone can further support previous findings and improve our understanding and recommendations for the management of these populations.

\section{Abbreviations}


HTN: systemic arterial hypertension; WHO: World Health Organization; SBP: systolic blood pressure; DBP: diastolic blood pressure; BP: blood pressure; RCTs: randomized controlled trials; PRISMA: Preferred Reporting Items for Systematic Reviews and Meta-analyses; PICOS: Population, Intervention, Comparison, Outcome, and Study; ABPM: ambulatory blood pressure monitoring; FINER: Feasible, Interesting, Novel, Ethical and Relevant; BMl: body mass index; GRADE: Grading of Recommendations Assessment, Development and Evaluation.

\section{Declarations}

- Ethics and systematic review registration: there are no major concern about ethical aspects for this study protocol. This protocol for the systematic review and meta-analysis was registered in the International Prospective Register of Systematic Reviews (PROSPERO) (www.crd.york.ac.uk/PROSPERO/, CRD 42020213081 and registration date 09/10/2020).

- Consent for publication: Not Applicable.

- Availability of data and material: Not Applicable.

- Competing interests: The authors declare that there are no competing interests.

- Funding: The authors received no financial support for the research, authorship, and/or publication of this article.

- Authors' contributions: PCO and GW were involved in conception and design of the study, data collection and data analysis plans, as well as drafting the manuscript. GW was involved in critical review of the article. AML was involved in writing, drafting and editing the final document for publication. Also, all authors have read and approved the manuscript.

- Acknowledgements: Authors thank to Carla Finger for her English assistance.

\section{References}

1. Mills KT, Bundy JD, Kelly TN, Reed JE, Kearney PM, Reynolds K, Chen J, He J. Global Disparities of Hypertension Prevalence and Control: A Systematic Analysis of Population-Based Studies From 90 Countries. Circulation. 2016;134:441-450. DOI: 10.1161/CIRCULATIONAHA.115.018912.

2. Forouzanfar MH, Liu P, Roth GA, Ng M, Biryukov S, Marczak L, Alexander L, Estep K, Hassen Abate K, Akinyemiju TF, et al. Global Burden of Hypertension and Systolic Blood Pressure of at Least 110 to 115 mm Hg, 1990-2015. JAMA. 2017;317:165-182. DOI: 10.1001/jama.2016.19043.

3. Kearney PM, Whelton M, Reynolds K, Muntner P, Whelton PK, He J. Global burden of hypertension: analysis of worldwide data. Lancet. 2005;365:217-223. DOI: 10.1016/S0140-6736(05)17741-1.

4. Lewington S, Clarke R, Qizilbash N, Peto R, Collins R, Collaboration PS. Age-specific relevance of usual blood pressure to vascular mortality: a meta-analysis of individual data for one million adults in 61 prospective studies. Lancet. 2002;360:1903-1913. DOI: 10.1016/s0140-6736(02)11911-8.

5. Lackland DT, Roccella EJ, Deutsch AF, Fornage M, George MG, Howard G, Kissela BM, Kittner SJ, Lichtman JH, Lisabeth LD, et al. Factors influencing the decline in stroke mortality: a statement from 
the American Heart Association/American Stroke Association. Stroke. 2014;45:315-353. DOI: 10.1161/01.str.0000437068.30550.cf.

6. Malta DC, Morais Neto OLd, Silva Junior JBd. Apresentação do plano de ações estratégicas para o enfrentamento das doenças crônicas não transmissíveis no Brasil, 2011 a 2022. Epidemiologia e Serviços de Saúde. 2011;20:425-438.

7. Malachias M, Gomes M, Nobre, Alessi, Feitosa AD, Coelho E. 7th Brazilian Guideline of Arterial Hypertension: Chapter 2 - Diagnosis and Classification. Arquivos Brasileiros de Cardiologia. 2016;107:7-13. DOI: 10.5935/abc.20160152.

8. Whelton PK, Carey RM, Aronow WS, Casey DE, Collins KJ, Dennison Himmelfarb C, DePalma SM, Gidding S, Jamerson KA, Jones DW, et al. 2017 ACC/AHA/AAPA/ABC/ACPM/AGS/APhA/ASH/ASPC/NMA/PCNA Guideline for the Prevention, Detection, Evaluation, and Management of High Blood Pressure in Adults: Executive Summary: A Report of the American College of Cardiology/American Heart Association Task Force on Clinical Practice Guidelines. Hypertension. 2018;71:1269-1324. DOI: 10.1161/HYP.0000000000000066.

9. Williams B, Mancia G, Spiering W, Agabiti Rosei E, Azizi M, Burnier M, Clement DL, Coca A, de Simone G, Dominiczak A, et al. 2018 ESC/ESH Guidelines for the management of arterial hypertension: The Task Force for the management of arterial hypertension of the European Society of Cardiology and the European Society of Hypertension: The Task Force for the management of arterial hypertension of the European Society of Cardiology and the European Society of Hypertension. J Hypertens. 2018;36:1953-2041. DOI: 10.1097/HJH.0000000000001940.

10. Owen A, Wiles J, Swaine I. Effect of isometric exercise on resting blood pressure: a meta analysis. J Hum Hypertens. 2010;24:796-800. DOI: 10.1038/jhh.2010.13.

11. Cornelissen VA, Fagard RH, Coeckelberghs E, Vanhees L. Impact of resistance training on blood pressure and other cardiovascular risk factors: a meta-analysis of randomized, controlled trials. Hypertension. 2011;58:950-958. DOI: 10.1161/HYPERTENSIONAHA.111.177071.

12. Cornelissen VA, Smart NA. Exercise training for blood pressure: a systematic review and metaanalysis. J Am Heart Assoc. 2013;2:e004473. DOI: 10.1161/JAHA.112.004473.

13. Inder JD, Carlson DJ, Dieberg G, McFarlane JR, Hess NC, Smart NA. Isometric exercise training for blood pressure management: a systematic review and meta-analysis to optimize benefit. Hypertens Res. 2016;39:88-94. DOI: 10.1038/hr.2015.111.

14. Jin YZ, Yan S, Yuan WX. Effect of isometric handgrip training on resting blood pressure in adults: a meta-analysis of randomized controlled trials. J Sports Med Phys Fitness. 2017;57:154-160. DOI: 10.23736/S0022-4707.16.05887-4.

15. Loaiza-Betancur AF, Chulvi-Medrano I. Is Low-Intensity Isometric Handgrip Exercise an Efficient Alternative in Lifestyle Blood Pressure Management? A Systematic Review. Sports Health. 2020;12:470-477. DOI: 10.1177/1941738120943882.

16. López-Valenciano A, Ruiz-Pérez I, Ayala F, Sánchez-Meca J, Vera-Garcia FJ. Updated systematic review and meta-analysis on the role of isometric resistance training for resting blood pressure 
management in adults. J Hypertens. 2019;37:1320-1333. DOI: 10.1097/HJH.0000000000002022.

17. Leung AA, Nerenberg K, Daskalopoulou SS, McBrien K, Zarnke KB, Dasgupta K, Cloutier L, Gelfer M, Lamarre-Cliche M, Milot A, et al. Hypertension Canada's 2016 Canadian Hypertension Education Program Guidelines for Blood Pressure Measurement, Diagnosis, Assessment of Risk, Prevention, and Treatment of Hypertension. Can J Cardiol. 2016;32:569-588. DOI: 10.1016/j.cjca.2016.02.066.

18. Brook RD, Appel LJ, Rubenfire M, Ogedegbe G, Bisognano JD, Elliott WJ, Fuchs FD, Hughes JW, Lackland DT, Staffileno BA, et al. Beyond medications and diet: alternative approaches to lowering blood pressure: a scientific statement from the american heart association. Hypertension. 2013;61:1360-1383. DOI: 10.1161/HYP.0b013e318293645f.

19. Carlson DJ, Dieberg G, Hess NC, Millar PJ, Smart NA. Isometric exercise training for blood pressure management: a systematic review and meta-analysis. Mayo Clin Proc. 2014;89:327-334. DOI: 10.1016/j.mayocp.2013.10.030.

20. De Souza Nery S, Gomides RS, da Silva GV, de Moraes Forjaz CL, Mion D, Tinucci T. Intra-arterial blood pressure response in hypertensive subjects during low- and high-intensity resistance exercise. Clinics (Sao Paulo). 2010;65:271-277. DOI: 10.1590/S1807-59322010000300006.

21. Narloch JA, Brandstater ME. Influence of breathing technique on arterial blood pressure during heavy weight lifting. Arch Phys Med Rehabil. 1995;76:457-462. DOI: 10.1016/s0003-9993(95)80578-8.

22. MacDougall JD, Tuxen D, Sale DG, Moroz JR, Sutton JR. Arterial blood pressure response to heavy resistance exercise. J Appl Physiol (1985). 1985;58:785-790. DOI: 10.1152/jappl.1985.58.3.785.

23. Carlson DJ, Inder J, Palanisamy SK, McFarlane JR, Dieberg G, Smart NA. The efficacy of isometric resistance training utilizing handgrip exercise for blood pressure management: A randomized trial. Medicine (Baltimore). 2016;95:e5791. DOI: 10.1097/MD.0000000000005791.

24. Pagonas N, Vlatsas S, Bauer F, Seibert FS, Zidek W, Babel N, Schlattmann P, Westhoff TH. Aerobic versus isometric handgrip exercise in hypertension: a randomized controlled trial. J Hypertens. 2017;35:2199-2206. DOI: 10.1097/HJH.0000000000001445.

25. McGowan CL, Visocchi A, Faulkner M, Verduyn R, Rakobowchuk M, Levy AS, McCartney N, MacDonald MJ. Isometric handgrip training improves local flow-mediated dilation in medicated hypertensives. Eur J Appl Physiol. 2007;99:227-234. DOI: 10.1007/s00421-006-0337-z.

26. Moher D, Shamseer L, Clarke M, Ghersi D, Liberati A, Petticrew M, Shekelle P, Stewart LA, Group P-P. Preferred reporting items for systematic review and meta-analysis protocols (PRISMA-P) 2015 statement. Syst Rev. 2015;4:1. DOI: 10.1186/2046-4053-4-1.

27. Higgins JPT, Cochrane Collaboration. Cochrane handbook for systematic reviews of interventions. Second edition. ed. Hoboken, NJ: Wiley-Blackwell; 2019. xxviii, 694 pages p.

28. Robinson KA, Dickersin K. Development of a highly sensitive search strategy for the retrieval of reports of controlled trials using PubMed. Int J Epidemiol. 2002;31:150-153. DOI: 10.1093/ije/31.1.150.

29. Glanville J, Foxlee R, Wisniewski S, Noel-Storr A, Edwards M, Dooley G. Translating the Cochrane EMBASE RCT filter from the Ovid interface to Embase.com: a case study. Health Info Libr J. 
2019;36:264-277. DOI: 10.1111/hir.12269.

30. Whiting P, Savović J, Higgins JP, Caldwell DM, Reeves BC, Shea B, Davies P, Kleijnen J, Churchill R, group R. ROBIS: A new tool to assess risk of bias in systematic reviews was developed. J Clin Epidemiol. 2016;69:225-234. DOI: 10.1016/j.jclinepi.2015.06.005.

31. Sterne JAC, Savović J, Page MJ, Elbers RG, Blencowe NS, Boutron I, Cates CJ, Cheng HY, Corbett MS, Eldridge SM, et al. RoB 2: a revised tool for assessing risk of bias in randomised trials. BMJ. 2019;366:I4898. DOI: 10.1136/bmj.14898.

32. Brasil. Ministério da Saúde. Secretaria de Ciência TelEDdCeTC-GdGdC. Diretrizes metodológicas: Sistema GRADE $\otimes$ manual de graduação da qualidade da evidência e força de recomendação para tomada de decisão em saúde. 2014. p. 71-71.

33. IntHout J, loannidis JP, Rovers MM, Goeman JJ. Plea for routinely presenting prediction intervals in meta-analysis. BMJ Open. 2016;6:e010247. DOI: 10.1136/bmjopen-2015-010247.

34. Dias S, Sutton AJ, Welton NJ, Ades AE. Evidence synthesis for decision making 3: heterogeneity-subgroups, meta-regression, bias, and bias-adjustment. Med Decis Making. 2013;33:618-640. DOI: $10.1177 / 0272989 \times 13485157$.

\section{Tables}




\section{Chart 1 - Search terms to be used to search Medline, EMBASE, Cochrane, LILACS and PEDro databases}

\section{MEDLINE (PubMed)}

Hypertension: (blood pressure, high OR blood pressures, high OR high blood pressure OR high blood pressures OR pre hypertension OR pre-hypertension OR pre hypertension OR prehypertension) AND

Exercise: (exercise OR exercises, isometric OR isometric exercises OR warm-up exercise OR exercise, warm-up OR exercises, warm-up OR warm up exercise OR warm-up exercises OR exercise, aerobic OR aerobic exercises OR exercises, aerobic OR aerobic exercise OR endurance, physical OR endurance, physical OR physical endurance OR training, resistance OR strength training OR training, strength OR weight-lifting OR strengthening program OR strengthening program, weight-lifting OR strengthening programs, weight-lifting OR weight lifting strengthening program OR weight-lifting strengthening programs OR weight-lifting exercise program OR exercise program, weight-lifting OR exercise programs, weight-lifting OR weight lifting exercise program OR weight-lifting exercise programs OR weight-bearing strengthening program OR strengthening program, weight-bearing OR strengthening programs, weight-bearing OR weight bearing strengthening program OR weight-bearing strengthening programs OR weight-bearing exercise program OR exercise program, weight-bearing OR exercise programs, weight-bearing OR weight bearing exercise program OR weight-bearing exercise programs OR activities, motor OR activity, motor OR motor activities OR physical activity OR activities, physical OR activity, physical OR physical activities OR locomotor activity OR activities, locomotor OR activity, locomotor OR locomotor activities) AND

Blood Pressure: (Pressure, Blood OR Diastolic Pressure OR Pressure, Diastolic OR Systolic Pressure OR Pressure, Systolic OR Pressures, Systolic OR Arterial Pressures OR Pressure, Arterial OR Pressures, Arterial OR Arterial Tension OR Arterial Tensions OR Tension, Arterial OR Tensions, Arterial OR Blood Pressure, Arterial OR Arterial Blood Pressure OR Arterial Blood Pressures OR Blood Pressures, Arterial OR Pressure, Arterial Blood OR Pressures, Arterial Blood OR Ambulatory Blood Pressure Monitoring OR Monitoring, Ambulatory Blood Pressure OR Blood Pressure Monitoring) AND

Type of study: (Randomized controlled trial[pt] OR controlled clinical trial[pt] OR randomized controlled trials[mh] OR random allocation[mh] OR double-blind method[mh] OR single-blind method[mh] OR clinical trial[pt] OR clinical trials[mh] OR ("clinical trial"[tw]) OR ((singl*[tw] OR doubl* [tw] OR trebl*[tw] OR tripl*[tw]) AND (mask*[tw] OR blind*[tw])) OR ("latin square"[tw]) OR placebos [mh] OR placebo*[tw] OR random*[tw] OR research design[mh:noexp] OR follow-up studies[mh] OR prospective studies[mh] OR cross-over studies[mh] OR control*[tw] OR prospectiv*[tw] OR volunteer*[tw])

\section{EMBASE}

Hypertension: ('hypertension'/exp OR hypertension OR 'prehypertension') AND

Exercise: ('exercise' OR 'aerobic exercise' OR 'isometric exercise' OR 'warm-up exercise' OR 'physical endurance' $O R$ 'endurance' OR 'training' OR 'resistance training' OR 'weight lifting' OR 'strengthening exercise' OR 'weight bearing' OR 'motor activity' OR 'physical activity') AND

Blood pressure: ('Blood Pressure' OR 'Diastolic Pressure' OR 'Systolic Pressure' OR 'Arterial Pressures' OR 'Arterial Tension' OR 'Arterial Blood Pressure' OR 'Ambulatory Blood Pressure Monitoring' OR 'Blood Pressure Monitoring') AND

Type of study: ('randomized controlled trial' OR 'controlled clinical trial')

\section{COCHRANE}

Hypertension: (blood pressure, high OR blood pressures, high OR high blood pressure OR high blood pressures OR pre hypertension OR pre-hypertension OR pre hypertension OR prehypertension) AND 
Exercise: (exercise $O R$ exercises, isometric $O R$ isometric exercises $O R$ warm-up exercise $O R$ exercise, warm-up OR exercises, warm-up OR warm up exercise OR warm-up exercises OR exercise, aerobic OR aerobic exercises $O R$ exercises, aerobic $O R$ aerobic exercise $O R$ endurance, physical $O R$ endurance, physical OR physical endurance OR training, resistance OR strength training OR training, strength $O R$ weight-lifting OR strengthening program OR strengthening program, weight-lifting OR strengthening programs, weight-lifting $O R$ weight lifting strengthening program $O R$ weight-lifting strengthening programs OR weight-lifting exercise program OR exercise program, weight-lifting OR exercise programs, weight-lifting OR weight lifting exercise program OR weight-lifting exercise programs $O R$ weight-bearing strengthening program OR strengthening program, weight-bearing OR strengthening programs, weight-bearing $O R$ weight bearing strengthening program OR weight-bearing strengthening programs $O R$ weight-bearing exercise program OR exercise program, weight-bearing OR exercise programs, weight-bearing $O R$ weight bearing exercise program OR weight-bearing exercise programs OR activities, motor OR activity, motor OR motor activities OR physical activity OR activities, physical OR activity, physical OR physical activities OR locomotor activity OR activities, locomotor OR activity, locomotor OR locomotor activities) AND

Blood Pressure: (Pressure, Blood OR Diastolic Pressure OR Pressure, Diastolic OR Systolic Pressure OR Pressure, Systolic OR Pressures, Systolic OR Arterial Pressures OR Pressure, Arterial OR Pressures, Arterial OR Arterial Tension OR Arterial Tensions OR Tension, Arterial OR Tensions, Arterial OR Blood Pressure, Arterial OR Arterial Blood Pressure OR Arterial Blood Pressures OR Blood Pressures, Arterial OR Pressure, Arterial Blood OR Pressures, Arterial Blood OR Ambulatory Blood Pressure Monitoring OR Monitoring, Ambulatory Blood Pressure OR Blood Pressure Monitoring)

\section{LILACS}

\section{LANGUAGE: Spanish}

Hipertensíon: (tw:(Hipertensión)) OR (tw:(Prehipertensión)) AND

Ejercicio: (tw:(Ejercicio Físico)) OR (tw:(Terapia por Ejercicio)) OR (tw:(Esfuerzo Físico)) OR (tw: (Aptitud Física)) OR (tw:(Actividad Motora)) AND (tw:(Presión Arterial)) AND

Presión arterial: (tw:(Monitoreo Ambulatorio de la Presión Arterial))

\section{LANGUAGE: Portuguese}

Hipertensão: (tw:(hipertensão)) OR (tw:(Pré-Hipertensão)) AND

Exercício: (tw:(Exercício Físico)) OR (tw:(Terapia por Exercício)) OR (tw:(Esforço Físico)) OR (tw: (Aptidão Física)) OR (tw:(Atividade Motora)) AND

Pressão arterial: (tw:(pressão arterial)) OR (tw:(Monitorização Ambulatorial da Pressão Arterial))

\section{LANGUAGE: English}

Hypertension: (tw:(hypertension)) OR (tw:(Prehypertension)) AND

Exercise: (tw:(exercise)) OR (tw:(Exercise Therapy)) OR (tw:(Physical Exertion)) OR (tw:(Physical Fitness)) OR (tw:(Motor Activity)) AND

Blood pressure: (tw:(Arterial Pressure)) OR (tw:(Blood Pressure Monitoring, Ambulatory))

\section{PEDro}

Exercise: Isometric exercise AND

Blood pressure: blood pressure 
OpenGrey: isometric exercise AND blood pressure

Banco de Teses e Dissertações CAPES*: exercício isométrico e pressão arterial em pré-hipertensos e hipertensos

ReBEC**: pressão arterial (search 1), exercício e pressão arterial (search 2)

ClinicalTrials**: hypertension OR prehypertension AND isometric exercise

WHO: hypertension OR prehypertension AND isometric exercise

* Filter: Grande Área Conhecimento (CIÊNCIAS DA SAÚDE), Área Avaliação (EDUCAÇÃO FÍSICA, ENFERMAGEM, MEDICINA I, MEDICINA II, MEDICINA III, NUTRIÇÃO)

** Filter: tipo de estudo (intervencional), Situação de recrutamento (Recrutando, Recrutamento concluído, Análise de dados complete)

*** Filter: Study type (interventional study (clinical trial))

\section{Figures}




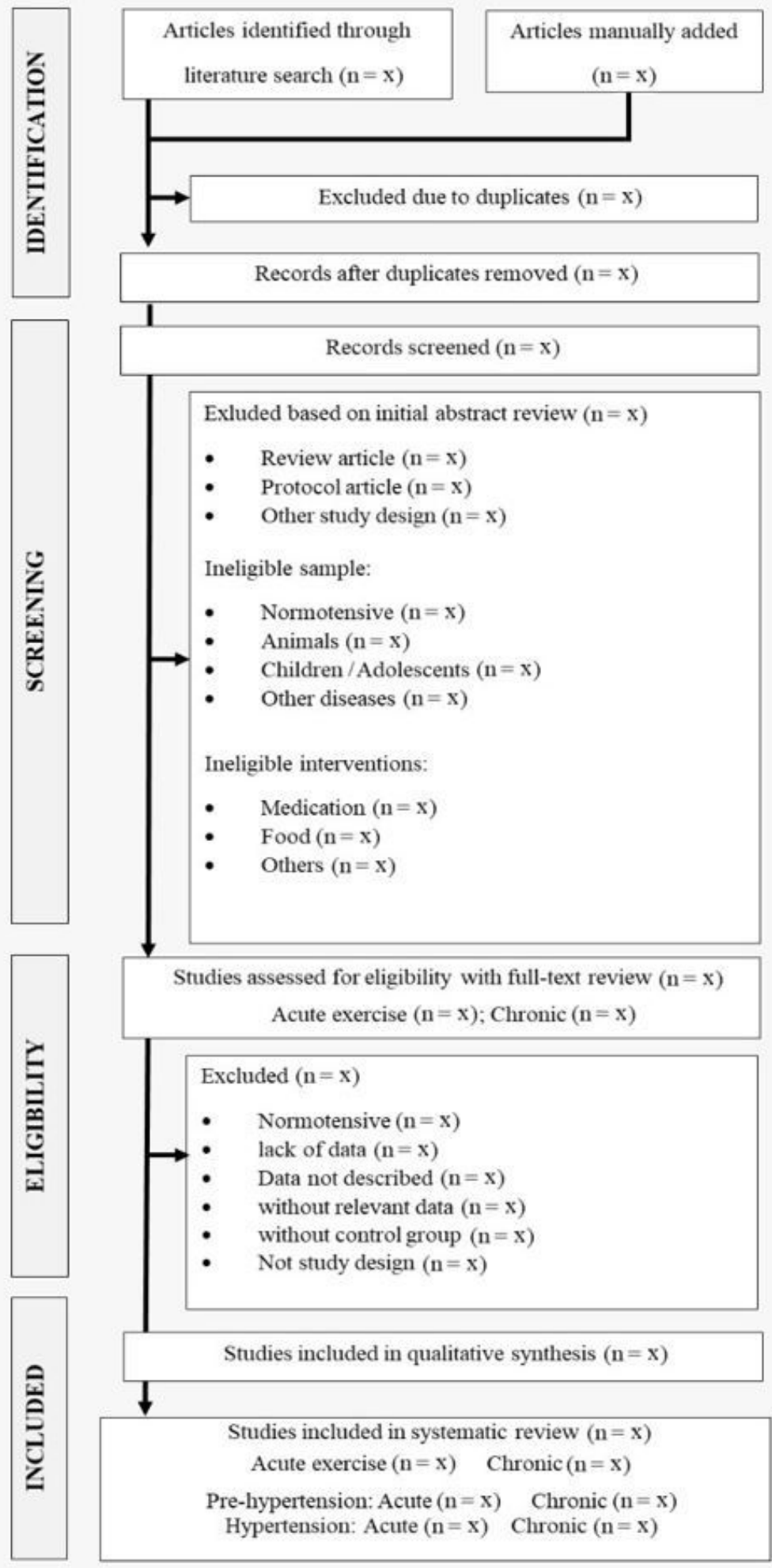

Figure 1

Flowchart of the systematic review study.

\section{Supplementary Files}

This is a list of supplementary files associated with this preprint. Click to download. 
- SupplementaryMaterial.docx

Page 16/16 